April 2018, Volume 3, Issue 2 (30-33)

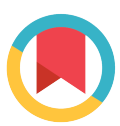

Check for updates

\title{
Determination of Arterial Compliance Using Thoracic Bioimpedance to Predict the Presence of Heart Diseases
}

\author{
Souhir Chabchoub ${ }^{1, *}$, Sofienne Mansouri ${ }^{1}$, Ridha Ben Salah ${ }^{2}$
}

${ }^{1}$ Higher Institute of Medical Technologies of Tunis, Laboratory of Biophysics and Medical Technologies, University of Tunis El Manar, Tunis,Tunisia

${ }^{2}$ College of Applied Medical Sciences, Salman Bin Abdulaziz University, Al-Kharj, Saudi Arabia

* Corresponding author: Souhir Chabchoub, PhD, Higher Institute of Medical Technologies of Tunis, Laboratory of Biophysics and Medical Technologies, University of

DOI: $10.21859 / \mathrm{ijcp}-03202$ Tunis El Manar, Tunis,Tunisia.E-mail: chabchoub_souhir@yahoo.fr

Submited: 01-04-2018

Accepted: 01-19-2018

Keywords:

Arterial compliance

Heart diseases

Thoracic bioimpedance.

(C) 2018. International Journal of Cardiovascular Practice.

\begin{abstract}
Introduction: Thoracic bioimpedance is a simple, cost-effective, and non-invasive tool used generally to determine several hemodynamic parameters. Based on this technique, the cardiac output and the stroke volume are the most common parameters used for diagnosing heart diseases. This study aims to investigate the ability of the arterial compliance parameter to predict or detect the presence of heart diseases.

Methods: two groups of young subjects participated in this study: a control group consisted of 10 subjects including 2 athletes and a group of 10 patients with various heart diseases. The thoracic bioimpedance recordings are used to determine the arterial compliance for the 2 groups of subjects. Statistical analyses are performed using the Student's t-test and the ROC curve analysis.

Results: Experimental results show that the arterial compliance is significantly lower in patients with heart diseases compared to control subjects $(\mathrm{P}<0.001)$.

Conclusions: The arterial compliance is a potentially useful hemodynamic parameter which could be successfully used to predict or detect the presence of heart diseases.
\end{abstract}

\section{INTRODUCTION}

Cardiovascular diseases (CVD) are the main cause of death in the world. The most important risk factors are hypertension, physical inactivity, smoking, unhealthy diet, high blood glucose, cholesterol and obesity. However, it has been reported that the early detection and the treatment of CVD can avoid any fatal complications and can consequently reduce the mortality rate $[1]$. Therefore, several screening tools have been used for diagnosing CVD such as Electrocardiography (ECG), Doppler Echocardiography, magnetic resonance imaging (MRI), chest X-ray, and etc. Biomedical researchers and scientists have always tried to find and develop the most effective and the easiest diagnostic tool. Particularly, thoracic bioimpedance is one of the screening techniques which are still under development. In fact, thoracic bioimpedance is a simple, non-invasive and cost-effective tool for monitoring electrical impedance changes in the thorax [2]. It has been used to compute various hemodynamic parameters such as cardiac output (CO), stroke volume (SV), left ventricular ejection time (LVET), arterial compliance, and etc. Based on these derived parameters, mainly the $\mathrm{CO}$ and the SV, bioimpedance has been widely used for diagnosing several heart diseases like mitral insufficiency [3] and myocardial infarction [4]. In this study, we proposed to use, precisely, the arte- rial compliance parameter to predict or detect the presence of heart diseases. Arterial compliance has not been extensively used for clinical screening purpose but it has been successfully used in other applications. Indeed, it has been used to evaluate the effects of aging on the arterial blood vessels; thus it has been reported that after the age 50 the arterial compliance decreases rapidly. Furthermore, this hemodynamic parameter has been considered as a marker of arterial stiffness and a clinical index of the buffering function of the arterial system. Besides, it has been demonstrated that an abnormalities in arterial compliance is associated to the presence of atherosclerotic plaque $[5,6]$. Clinically, arterial compliance is defined as the change in arterial blood volume $(\Delta \mathrm{V})$ due to a given change in arterial blood pressure $(\Delta \mathrm{P})$. Precisely, arterial compliance is described as the ratio between the volume variation and the pressure variation $(\Delta \mathrm{V} / \Delta \mathrm{P})$. Therefore, a high blood pressure (hypertension) can be due to a decrease in arterial compliance [5]. As mentioned above, hypertension has been considered as one of the most important cardiovascular risk factor, thus according to our point of view, the arterial compliance can be used as a predictor of a future heart disease. The above theoretical estimate will be developed and discussed in this current study. 


\section{METHODS}

\section{Study Population}

For this study, inclusion criteria were as follows: (1) an age less than 35 years and more than 18 years, (2) patients with isolated heart disease (the patients who had additional diseases were excluded), (3) absence of an artificial pacemaker, (4) male gender, (5) non-smokers population. The study population consisted of two groups of subjects: a control group of 10 subjects including 2 athletes ( $100 \%$ male, having an average age of $25.6 \pm 5.17$ years: ranging from 18 to 34 years) and a group of 10 patients with various heart diseases, including mitral stenosis, cardiac insufficiency and myocardial infarction ( $100 \%$ male, having an average age of $25.9 \pm 4.36$ years: ranging from 21 to 32 years).

\section{Thoracic Bioimpedance Measurements}

The thoracic bioimpedance recordings used in this study were obtained following the method of Ben Salah et al. [7]. In this method, a constant, alternating, high frequency, and small current $(1 \mathrm{~mA}, 30 \mathrm{kHz})$ is applied, through two electrodes, into the forehead and above the leading edge of the heart. Two other electrodes are placed between the injection electrodes (in the left side of the patients' chest), at the level of aorta 2 or $3 \mathrm{~cm}$ apart, to measure the acquired voltage. During the signal recordings, the patient must lie supine, relaxed and in expiratory apnea for a period of 10 seconds. The proposed electrodes configuration is shown in Fig 1.

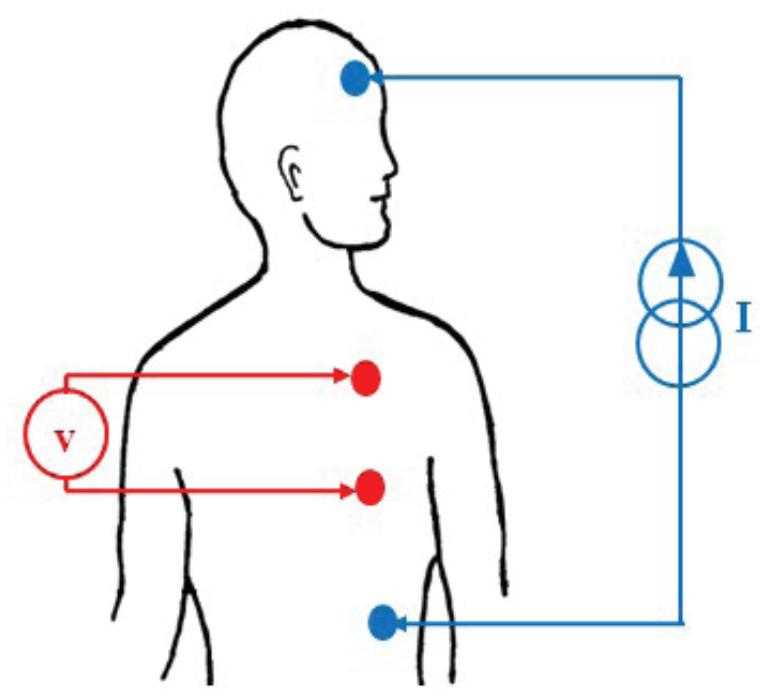

Figure 1: Electrodes Configuration for the Thoracic Bioimpedance Measurement

The bioimpedance recording represents the impedance variation of the explored thoracic region versus time samples. A typical thoracic bioimpedance tracing is shown in Fig 2. Mathematically, the impedance $(\mathrm{Z})$ is defined by Ohm's law as the ratio between the voltage signal measured (V) and the current signal injected (I). The impedance is inversely proportional to the chest's fluid content; it increases or decreases when the fluid falls or rises, respectively. The impedance varies with tissue composition, tissue structure and tissue health status [8].

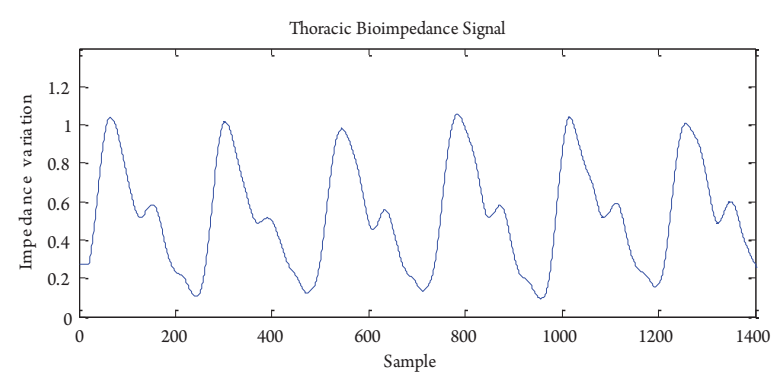

Figure 2: Typical Example of Thoracic Bioimpedance Tracing

\section{The Determination of Arterial Compliance}

The first purpose of this current study is the determination of the arterial compliance using the thoracic bioimpedance technique. The second purpose consists to investigate the ability of this estimated compliance in the prediction or the detection of heart diseases.

As mentioned above the arterial compliance $\mathrm{C}_{\mathrm{p}}$ is the ratio of arterial blood volume $\Delta \mathrm{V}$ to arterial blood pressure $\Delta \mathrm{P}$ :
$\mathrm{C}_{\mathrm{p}}=\Delta \mathrm{V} / \Delta \mathrm{P}$
Eq. 1

Using the thoracic bioimpedance, the pulsatile volume change is presented as follows:

$\Delta \mathrm{V}=\left(\rho \mathrm{L}^{2} \Delta \mathrm{Z}\right) /\left(\mathrm{Z}_{0}^{2}\right) \quad$ Eq. 2

Where:

$\rho$ : is the resistivity of blood $(\Omega . \mathrm{cm})$.

$\mathrm{L}:$ is the length of the explored section $(\mathrm{cm})$.

$\Delta \mathrm{Z}$ : is the magnitude of the pulsatile impedance change (impedance variation).

$\mathrm{Z}_{0}$ : is the impedance measured when the pulsatile volume is minimum (diastolic or basic impedance) $(\Omega)$.

The instantaneous aortic pressure $\mathrm{P}(\mathrm{t})$ derived from the thoracic bioimpedance record was determined using the mathematical formula developed in [9]:

$\mathrm{P}(\mathrm{t})=\mathrm{P}_{\text {Dia }}+\left(\mathrm{P}_{\text {Sys }}-\mathrm{P}_{\text {Dia }}\right)(\mathrm{Z}(\mathrm{t})) / \mathrm{Z}_{\text {max }} \quad$ Eq.3

Where:

$\mathrm{P}_{\text {Sys }}$ : presents the systolic blood pressure $(\mathrm{mmHg})$.

$\mathrm{P}_{\mathrm{Dia}}$ : presents the diastolic blood pressure $(\mathrm{mmHg})$.

$\mathrm{Z}(\mathrm{t})$ : is the instantaneous bioimpedance signal.

$\mathrm{Z}_{\max }$ : is the maximum amplitude of the thoracic bioimpedance record.

Consequently, the arterial compliance $\mathrm{C}_{\mathrm{p}}(\mathrm{ml} / \mathrm{mmHg})$ was estimated as follows [10]:

$\mathrm{C}_{\mathrm{p}}=\left(\rho \mathrm{L}^{2} \mathrm{Z}_{\text {max }}\right) /\left(\mathrm{Z}_{0}^{2}\left(\mathrm{P}_{\text {Sys }}-\mathrm{P}_{\text {Dia }}\right)\right) \quad$ Eq.4

Noted that the systolic and the diastolic blood pressures $\left(\mathrm{P}_{\text {Sys }}\right.$ and $\left.\mathrm{P}_{\mathrm{Dia}}\right)$ were non-invasively measured using an electronic sphygmomanometer model, MP3 FUZZY.

Throughout this study, the arterial compliance $\mathrm{C}_{\mathrm{p}}$ is determined for 2 groups of subjects (control subjects and patients having various heart diseases) in order to evaluate the capacity of this hemodynamic parameter $\left(\mathrm{C}_{\mathrm{p}}\right)$ to predict or detect the presence of heart diseases.

\section{Statistical Analysis}

In this study, data were expressed as mean \pm standard deviation (SD). Student's t-test was used to evaluate the difference in arterial compliance values between two groups of subjects (control subjects and patients with various heart diseases). Moreover, the Receiver Operating Characteristic (ROC) 
curve was used to evaluate the ability of the arterial compliance to distinguish between control subjects and patients with heart diseases. The optimum cut-off, sensitivity (Se), specificity (Sp), negative predictive values (NPV), positive predictive values (PPV), and the area under the ROC curve (AUC) were computed. The ROC curve was generated using the MedCalc software, version 17.8. Besides, a p-value of less than 0.05 was considered statistically significant.

\section{RESULTS}

The estimated arterial compliance values for control subjects and patients with various heart diseases are shown in Fig 3. In terms of this figure, it is clearly seen that the arterial compliance is lower in patients with heart diseases (blue graph: from patients D1 to patients D10) compared to control subjects (red graph: from subjects $\mathrm{C} 1$ to subjects CA10). Particularly, for control subjects, CA9 and CA10 subjects have the highest arterial compliance values; indeed CA9 and CA10 correspond to 2 athlete subjects.

The mean value $( \pm S D)$ of the arterial compliance for control subjects and patients with various heart diseases is illustrated in Table 1. It is vivid from this table that the arterial compliance was significantly decreased in patients with heart diseases compared to control subjects $(2.11 \pm 0.79 \mathrm{ml} / \mathrm{mmHg}$ for control subjects vs. $0.99 \pm 0.14 \mathrm{ml} / \mathrm{mmHg}$ for patients with heart diseases). Student's t-test demonstrates that there was highly significant difference between control subjects and patients with heart diseases $(\mathrm{P}<0.001)$.

The ROC curve analysis results of the arterial compliance parameter are illustrated in Table 2. The optimum cut-off is lower than, or equal to, $1.18 \mathrm{ml} / \mathrm{mmHg}$, the sensitivity, the specificity, the positive predictive value, and the negative predictive values are about $100 \%$. The AUC of the ROC curve is equal to 1 . Therefore, the obtained results demonstrate the high ability of the arterial compliance to distinguish between control subjects and patient with heart diseases $(\mathrm{P}<0.0001)$. The ROC curve of the arterial compliance is shown in Fig 4.

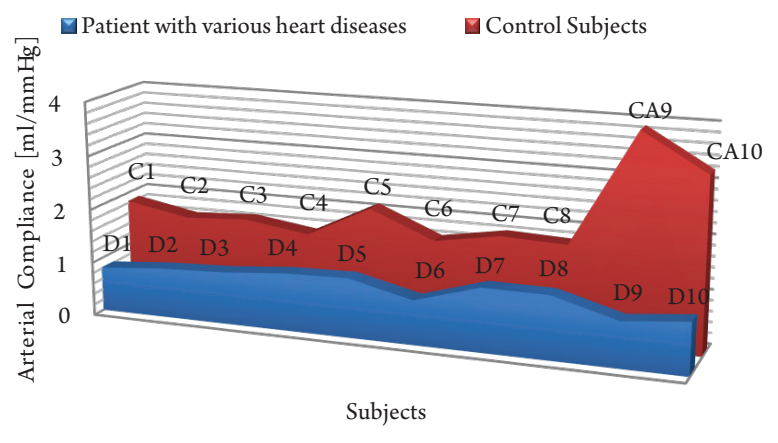

Figure 3: The Estimated Arterial Compliance for Control Subjects (Red Graph: from Subject C1 to Subject CA10) and Patients with Heart Diseases (Blue Graph: from Patient D1 to Patient D10).

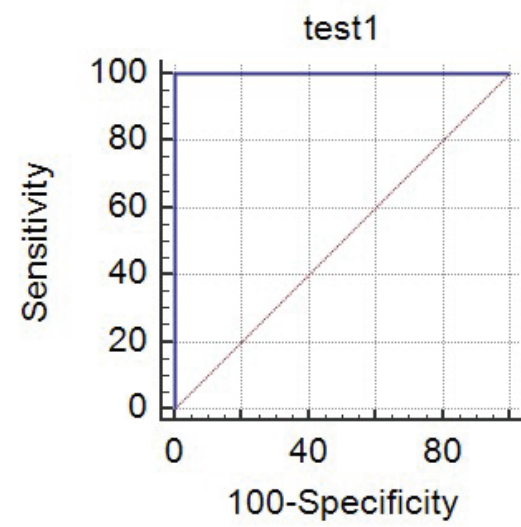

Figure 4: ROC Curve for the Arterial Compliance Parameter $(\mathrm{AUC}=1, \mathrm{P}<0.0001)$.

Table 1: Mean Value ( \pm SD) of the Arterial Compliance for Control Subjects and Patients with Various Heart Diseases

\begin{tabular}{lccc} 
Hemodynamic parameter & Control subjects & Patients with various heart diseases & $t$-values \\
Arterial Compliance $(\mathrm{ml} / \mathrm{mmHg})$ & $2.11 \pm 0.79$ & $0.99 \pm 0.14$ & $4.17^{*}$ \\
\hline
\end{tabular}

Note: ${ }^{*} \mathrm{P}<0.001$ : The Difference is Highly Significant.

Table 2: ROC Curve Analysis of the Arterial Compliance Parameter.

\begin{tabular}{lccccccc} 
Hemodynamic parameter & AUC & $\mathrm{P}$ & Optimum cut-off & Se $(\%)$ & Sp $(\%)$ & PPV $(\%)$ & NPV $(\%)$ \\
Arterial Compliance & 1 & $<0.0001$ & $\leq 1.18 \mathrm{ml} / \mathrm{mmHg}$ & 100 & 100 & 100 & 100 \\
\hline
\end{tabular}

Note: AUC: Area Under The ROC Curve; Se: Sensitivity; Sp: Specificity; PPV: Positive Predictive Value; NPV: Negative Predictive Value

\section{DISCUSSION}

The main purpose of this study is to investigate the ability of arterial compliance to predict or detect the presence of heart diseases. This important hemodynamic parameter is estimated using the thoracic bioimpedance technique which still under development. In order to avoid the effects of age in arterial compliance, we have included only young subjects. The high blood pressure is considered as the most important risk factor for cardiovascular diseases; this cause a theoretical falls in the atrial compliance. In this study, experimental results demonstrate that the arterial compliance is significantly lower in patients with heart diseases than in control subjects. These results present a high degree of reproducibility for all the study population. Therefore, we can assume that the arterial compliance is a useful and efficient hemodynamic parameter that can be used to detect the presence of heart diseases. Moreover, arterial compliance can be used as a predictor of a 
future heart disease. On the other hand, the obtained results also show that the arterial compliance increases in athlete subjects. Thus, we can report that the arterial compliance can distinguish between normal and athlete subjects. However, the lack of a "gold standard" tool for estimating arterial compliance remains a major drawback in this study. Usually, the arterial compliance can be estimated using a variety of techniques like angiography, magnetic resonance imaging, transthoracic echocardiography [5], and etc. Although the accuracy of these techniques, they are still expensive, discontinuous and require high level skills. For these reasons, we suggested in this current study to use the thoracic bioimpedance technique which is simple, low-cost, continuous (can perform an ambulatory measurement) and requires less skill. In the future research, we will try to use another tool for the determination of arterial compliance (like the magnetic resonance imaging MRI) in order to perform a comparison between the obtained results. Moreover, a larger number of patients are needed in order to further quantify and recommend our method. Furthermore, we will investigate the ability of the arterial compliance parameter to distinguish between several heart diseases.

\section{AUTHORS' CONTRIBUTION}

Conception or design of the work: Souhir, Sofienne, Ridha Data collection: Ridha

Data analysis and interpretation: Ridha, Souhir

Drafting the article: Souhir, Sofienne

Critical revision of the article: Souhir

Final approval of the version to be published: Souhir, Sofienne

\section{CONFLICTS OF INTEREST}

The authors declare that there is no conflict of interest regarding the publication of this paper.

\section{REFERENCES}

1. Benjamin EJ, Blaha MJ, Chiuve SE, Cushman M, Das SR, Deo R, et al. Heart Disease and Stroke Statistics-2017 Update: A Report From the American Heart Association. Circulation. 2017; 135(10):e146-e603. DOI: $10.1161 /$ CIR.0000000000000485 PMID: 28122885

2. Patterson RP. Impedance cardiography: What is the source of the signal? J Phys. 2010;224:012118. DOI: 10.1088/1742$6596 / 224 / 1 / 012118$

3. Chabchoub S, Mansouri S, Ben salah R. Diagnosis of mitral insufficiency using Impedance Cardiography Technique ICG. J Electr Bioimped. 2016;7(1):28. DOI: $10.5617 /$ jeb.2872

4. Chen SJ, Gong Z, Duan QL. Evaluation of heart function with impedance cardiography in acute myocardial infarction patients. Int $\mathrm{J}$ Clin Exp Med. 2014;7(3):719-27. PMID: 24753769

5. McVeigh GE, Bank AJ, Cohn JN. Arterial Compliance. In: Willerson J, Cohn J, editors. Cardiovascular medicine. London: Springer 2007. p. 1811-31.

6. Glasser SP, Arnett DK, McVeigh GE, Finkelstein SM, Bank AJ, Morgan DJ, et al. Vascular compliance and cardiovascular disease: a risk factor or a marker? Am J Hypertens. 1997;10(10 Pt 1):1175-89. DOI: 10.1016/s0895-7061(97)00311-7 PMID: 9370391

7. Salah RB, Marrakchi A, Ellouze N. Cardiac diseases quantification of by temporal and cepstral analysis of plethysmographic signal. Med J Islamic World Acad Sci. 1989;2(3):204-11.

8. Bera TK. Bioelectrical Impedance Methods for Noninvasive Health Monitoring: A Review. J Med Eng. 2014;2014:381251. DOI: 10.1155/2014/381251 PMID: 27006932

9. Mansouri S, Mahjoubi H, Salah RB. Determination of instantaneous arterial blood pressure from bio-impedance signal. J Biophys Struct Biol. 2010;2(1):009-15.

10. Mansouri S, Mahjoubi H, Salah RB. Conception d'un prototype de bio-impédance périphérique autour d'un FPGA Virtex-5 LX30. IRBM. 2010;31(5-6):280-8. 